\title{
Disordered one-dimensional Bose-Fermi mixtures: The Bose-Fermi glass
}

\author{
François Crépin, ${ }^{1}$ Gergely Zaránd, ${ }^{2}$ and Pascal Simon ${ }^{1}$ \\ ${ }^{1}$ Laboratoire de Physique des Solides, CNRS UMR-8502 Université Paris Sud, 91405 Orsay Cedex, France \\ ${ }^{2}$ Budapest University of Technology and Economics, H-1521 Budapest, Hungary
}

(Dated: October 17, 2018)

\begin{abstract}
We analyze an interacting Bose-Fermi mixture in a 1D disordered potential using a combination of renormalization group and variational methods. We obtain the complete phase diagram in the incommensurate case as a function of bosonic and inter-species interaction strengths, in the weak disorder limit. We find that the system is characterized by several phase transitions between superfluid and various glassy insulating states, including a new Bose-Fermi glass phase, where both species are coupled and localized. We show that the dynamical structure factor, as measured through Bragg scattering experiments, can distinguish between the various localized phases and probe their dynamics.
\end{abstract}

PACS numbers: 67.60.Fp, 67.85.Pq, 71.10.Pm, 71.23.-k, 71.55.Jv

Introduction. Spectacular developments of experimental techniques in the field of ultra-cold atoms have opened a new way in the study of strongly correlated systems [1]. Experimentalists are now able to create optical lattices with highly tunable parameters, and realize various models and quantum phase transitions. In Ref. [2], e.g., superfluid bosons were driven to a Mott insulating state, by tuning the on-site interactions of the Hubbard model, and the incompressibility of this state has also been demonstrated [3]. The Mott transition was also realized in Fermi systems [4], where other interesting phenomena such as pairing with spin imbalance [5, 6] or the BCS-BEC crossover [1] were also investigated.

Having more and more control over conventional systems, experimentalists and theorists now turn to the study of more complicated ones. On the one hand, a lot of attention is devoted to multicomponent systems such as Bose-Fermi mixtures [7-11], or three component systems [12], which offer the possibility of realizing new phases of matter, such as supersolids, color superconductivity [13], or 'baryonic' phases [14]. On the other hand, the creation of disorder using speckle lasers or incommensurate laser beams in trapped systems paved the road to create quantum glasses and Anderson insulators in cold atomic systems [15, 16].

It is well known that impurities can lead to the localization of electronic wave functions. While interactions are known to be important in the localized phase, taking them into account is quite a difficult task. In one dimension, this question has been addressed both for fermions [17] and for bosons [17, 18]. For spinless fermions, sufficiently attractive interactions trigger a transition from the Anderson insulating phase to a p-wave superconducting phase. For bosons, a disorder-induced insulating phase appears for sufficiently strong interactions: nicknamed Bose glass, it has no gap, it is insulating, and is compressible [18]. The Bose glass disappears at sufficiently small repulsive interactions, where the system enters a superfluid phase. On the other hand, for even

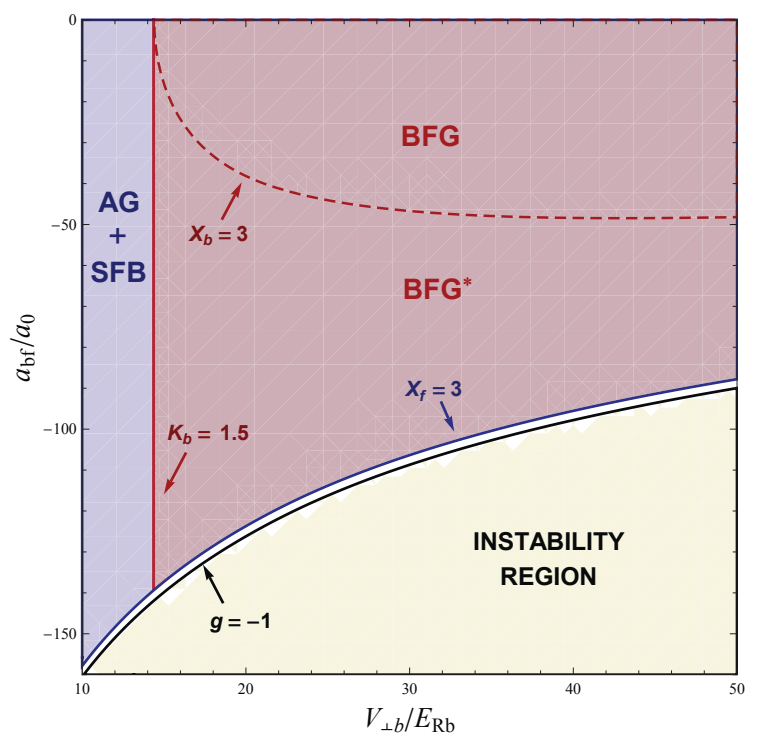

FIG. 1: Phase diagram of a $1 \mathrm{D}{ }^{87} \mathrm{Rb}^{-}{ }^{40} \mathrm{~K}$ Bose-Fermi mixture, in the weak disorder limit. We consider an array of tubes, created with lasers of wavelength $\lambda=1064 \mathrm{~nm}$, which corresponds to a (2D) lattice constant $d=\lambda / 2$. We take $a_{b b}=$ $100 a_{0}$ as Bose-Bose scattering length, while the Bose-Fermi scattering length, $a_{b f}$, is tuned using a Feshbach resonance. The one.dimensional densities are chosen to be $\rho_{f} d=0.3$ and $\rho_{b} d=0.1$. The recoil energy is $E_{R, b}=h^{2} \lambda^{-2} /\left(2 m_{R b}\right)$, while $V_{\perp b}$ is the transverse confining potential creating the $1 \mathrm{D}$ tubes. The Bose-Bose interaction $U_{b}$ increases with $V_{\perp b}$ [30]. Four phases and the region of instability of the Luttinger liquid theory are shown. BFG: Bose-Fermi glass, BFG* (BFG with an extremely large bosonic localization length), AG+SFB: Anderson Glass + Superfluid Bosons, LL: Luttinger liquid (above the instability region) .

smaller interactions bosons should undergo Anderson localization and condense in the lowest eigenstate [17, 18]. The Bose glass [19] and the Anderson glass [15] phases were now observed experimentally.

In the present paper we wish study, how the most basic 
two-component system, the Bose-Fermi mixture (BFM) is affected by the presence of disorder. This is not only important in view of the current experimental interest [7-11], but it is a challenging fundamental question on its own, too, especially in the limit of strong interactions. In particular, one may wonder whether disorder "decouples" both species or whether the localization of one species triggers the localization of the other species because of interactions. This issue has been discussed in a general context in Ref. [20], where a variety of exotic situations were considered. In this letter we focus on a disordered one-dimensional (1D) BFM, where interactions are particularly relevant. One-dimensional BFMs in the absence of disorder have been investigated by a variety of analytical [21, 22] and numerical [23] techniques. Here, we follow Refs. [17, 18] and use bosonization to extract the low-energy properties of the BFM in a disordered potential. We focus on the case where the bosonic and fermionic densities are not commensurate either with the lattice constant or with each other. Our main results are summarized for a ${ }^{87} \mathrm{Rb}^{-40} \mathrm{~K}$ mixture in Fig. 11. We find two different glassy phases as well as a Luttinger liquid phase and a region where the Luttinger liquid theory breaks down (see caption).

Model. We consider a 1D mixture of bosons and spinless fermions described by the following Hamiltonian, $H=H_{b}+H_{f}+H_{b f}+H_{\text {dis }}$, where

$$
\begin{aligned}
H_{\alpha} & =\int d x\left[-\psi_{\alpha}^{\dagger}(x) \frac{\partial_{x}^{2}}{2 m_{\alpha}} \psi_{\alpha}(x)+\frac{U_{\alpha}}{2}: \rho_{\alpha}^{2}(x):\right] \\
H_{b f} & =U_{b f} \int d x \delta \rho_{b}(x) \delta \rho_{f}(x) \\
H_{\text {dis }} & =\int d x\left(V_{b}(x) \rho_{b}(x)+V_{f}(x) \rho_{f}(x)\right) .
\end{aligned}
$$

Here $\psi_{\alpha}(\alpha=b, f)$ denote the Bose/Fermi fields, $\rho_{\alpha}(x)=$ $\psi_{\alpha}^{\dagger}(x) \psi_{\alpha}(x)$ their density, and $m_{\alpha}$ the mass of the bosons/fermions. The symbol : $\cdots$ : stands for normal ordering. The interaction between bosons must be repulsive for stability reasons, $U_{b}>0$, while for spinless fermions with a local interaction, $U_{f} \equiv 0$. Bosons and fermions can have either repulsive $\left(U_{b f}>0\right)$ or attractive $\left(U_{b f}<0\right)$ interactions. The random potentials, $V_{f / b}(x)$ describe the effects of disorder. For simplicity, here we assume them to be Gaussian with zero mean values, and spatially uncorrelated, i.e., $\overline{V_{\alpha}(x) V_{\alpha}\left(x^{\prime}\right)}=D_{\alpha} \delta\left(x-x^{\prime}\right)$ [31]. Experimentally, there is only one disorder potential, for instance a laser speckle. As a result, $V_{f}$ and $V_{b}$ are correlated [32]. Nevertheless, $\rho_{b}$ being incommensurate with $\rho_{f}$, we find that for long wavelength excitations $V_{b}$ and $V_{f}$ act as if they were independent [28]. In the following, we therefore assume $\overline{V_{f}(x) V_{b}\left(x^{\prime}\right)}=0$.

In $1 \mathrm{D}$, long wavelength excitations are essentially density fluctuations, and the densities $\varrho_{f / b}$ and the fields $\psi_{f / b}$ can be "bosonized", i.e., represented in terms of bosonic phases, $\phi_{f / b}$ [24]. Then $H_{f}$ and $H_{b}$ take on simple quadratic forms

$$
H_{\alpha}=\frac{v_{\alpha}}{2 \pi} \int d x\left[K_{\alpha}\left(\partial_{x} \theta_{\alpha}(x)\right)^{2}+\frac{1}{K_{\alpha}}\left(\partial_{x} \phi_{\alpha}(x)\right)^{2}\right],
$$

with $\phi_{\alpha}(x), \theta_{\alpha}(x)$ denoting density and phase quantum fields, obeying $\left[\phi_{\alpha}\left(x^{\prime}\right), \partial_{x} \theta_{\alpha}(x)\right]=i \pi \delta\left(x-x^{\prime}\right)$. The prefactor $v_{b}$ can be viewed as the sound velocity for Bogoliubov phonons in a quasi-condensate, while $v_{f}$ is the Fermi velocity. The dimensionless Luttinger parameters, $K_{f}$ and $K_{b}$, characterize the strength of interactions [25]: In our case of non-interacting fermions, $K_{f}=1$. For bosons, $K_{b}=1$ in the hardcore limit, $K_{b}>1$ for softer repulsive interactions, and $K_{b} \rightarrow \infty$ for non-interacting bosons. Keeping in mind that $\varrho_{b}$ is incommensurate with $\varrho_{f}$, backward scattering of bosons on fermions is irrelevant, and at low energies $H_{b f}$ can be approximated as

$$
H_{b f}=\frac{U_{b f}}{\pi^{2}} \int d x \partial_{x} \phi_{b}(x) \partial_{x} \phi_{f}(x) \text {. }
$$

In the same way, disorder induces backward scattering and forward scattering. However, the forward scattering can be gauged away (without affecting current-current and superfluid correlations), and only backward scattering responsible for localization remains [17, 28],

$$
H_{d i s}^{\alpha}=\int d x\left[\rho_{\alpha} \xi_{\alpha}(x) e^{-i 2 \phi_{\alpha}(x)}+h . c .\right]
$$

with $\overline{\xi_{\alpha}(x) \xi_{\alpha}^{*}\left(x^{\prime}\right)}=D_{\alpha} \delta\left(x-x^{\prime}\right)$.

Renormalization group (RG). To perform the RG analysis, we use the replica trick. We introduce $n$ replicas of the system, average over disorder, and then take $n \rightarrow 0$. The replicated action then reads $S_{\text {rep }}=S_{0}+S_{\text {dis }}^{\text {rep }}$, with

$$
\begin{aligned}
S_{0} & =\sum_{a=1}^{n} \sum_{\alpha=f, b} \frac{1}{2 \pi K_{\alpha}} \int d x d \tau\left[\frac{1}{v_{\alpha}}\left(\partial_{\tau} \phi_{\alpha}^{a}\right)^{2}+v_{\alpha}\left(\partial_{x} \phi_{\alpha}^{a}\right)^{2}\right]+\sum_{a=1}^{n} \frac{U_{b f}}{\pi^{2}} \int d x d \tau \partial_{x} \phi_{f}^{a} \partial_{x} \phi_{b}^{a}, \\
S_{\mathrm{dis}}^{\mathrm{rep}} & =-D_{f} \rho_{f}^{2} \sum_{a, b} \int d x d \tau d \tau^{\prime} \cos \left(2 \phi_{f}^{a}(x, \tau)-2 \phi_{f}^{b}\left(x, \tau^{\prime}\right)\right)-D_{b} \rho_{b}^{2} \sum_{a, b} \int d x d \tau d \tau^{\prime} \cos \left(2 \phi_{b}^{a}(x, \tau)-2 \phi_{b}^{b}\left(x, \tau^{\prime}\right)\right) .
\end{aligned}
$$

Integrating out high momentum degrees of freedom we

find the following flow equations to lowest order,

$$
\begin{aligned}
\frac{d \tilde{D}_{\alpha}}{d l} & =\left(3-X_{\alpha}\right) \tilde{D}_{\alpha}(l) \\
\frac{d}{d l}\left(K_{\alpha} v_{\alpha}\right) & =-2 K_{\alpha}(l) v_{\alpha}(l) \tilde{D}_{\alpha}(l) \mathcal{C}_{\alpha}
\end{aligned}
$$


while $\frac{d}{d l}\left(K_{\alpha} / v_{\alpha}\right)=0$. Here we have defined dimensionless variables for the disorder, $\tilde{D}_{\alpha}=\frac{2}{\pi \Lambda_{0}} \frac{K_{\alpha}^{2}}{v_{\alpha}^{2}} D_{\alpha}$, and parametrized the high-energy cut-off as $\Lambda=\Lambda_{0} e^{-l}$. The $\mathcal{C}_{\alpha}$ are numbers coming from our RG scheme. The anomalous dimensions, $X_{f / b}$, of the disorder operators can be obtained from the diagonalization of $S_{0}$ and read:

$$
X_{f}=\frac{2 K_{f}\left(1+t \sqrt{1-g^{2}}\right)}{\sqrt{1-g^{2}} \sqrt{1+2 t \sqrt{1-g^{2}}+t^{2}}},
$$

where $t=v_{f} / v_{b}$ and $g=U_{b f} / \pi \sqrt{K_{f} K_{b} /\left(v_{f} v_{b}\right)}$ is a dimensionless parameter. The dimension $X_{b}$ is obtained by changing $t \rightarrow 1 / t$ and $K_{f}$ into $K_{b}$ in Eq (10).

For uncoupled species $(g=0), X_{f}=2 K_{f}$ and $X_{b}=$ $2 K_{b}$. Thus spinless fermions (bosons) are localized when $K_{f}<3 / 2\left(K_{b}<3 / 2\right)$ [17]. At weak disorder we can neglect the feed-back, Eq. (9), and follow, e.g. Ref. 25, to relate $K_{f}, K_{b}, t, g$ to experimental parameters, and construct the phase diagram of Fig. 11 The thick solid blue line denotes $X_{f}=3$, while the dashed red line indicates $X_{b}=3$. Naively, one would expect these lines to separate three phases: In the Bose-Fermi glass phase (BFG) $X_{b}<3$ and $X_{f}<3$, both species are pinned by disorder, and fermionic and bosonic excitations are localized over the localization lengths, $\xi_{f}$ and $\xi_{b}$, respectively. In the Luttinger liquid (LL) phase $X_{b}>3$ and $X_{f}>3$, disorder is thus irrelevant, and both species are superfluid. In the regime, $X_{b}>3$ and $X_{f}<3$ one would naively predict a phase with localized fermions forming an Anderson glass (AG) and superfluid bosons (SFB). In this regime, however, some care needs be taken: since fermions are localized, fermionic density fluctuations become "gapped" at a length scale $\Lambda \sim 1 / \xi_{f}$. Below this scale bosons interact with their bare interaction. Thus the RG equations become

$$
\frac{d \log \tilde{D}_{b}}{d l}= \begin{cases}3-X_{b} & \text { if } \Lambda \gg 1 / \xi_{f} \\ 3-2 K_{b} & \text { if } \Lambda \ll 1 / \xi_{f}\end{cases}
$$

As a consequence, the region $X_{b}>3$ and $X_{f}<3$ is divided into two phases. In the region, $X_{f}<3$ and $K_{b}>3 / 2$, we obtain an $\mathrm{AG}+\mathrm{SFB}$ phase. However, in the region $X_{f}<3$ and $K_{b}<3 / 2$ bosons are ultimately localized on a very large length scale, and we find a BoseFermi glass phase (BFG*).

To support the RG picture and to describe the "gapped" phases, we made use of the Gaussian variational method (GVM) in replica space, as introduced in [26] and used in Ref. 27] to treat interacting 1D disordered media. This method is able to capture the localized compressible phases. To start with, we rewrite the action $S_{0}$ in Fourier space as

$$
S^{0}=\frac{1}{2} \frac{1}{\beta L} \sum_{q, i \omega_{n}} \phi_{\alpha}^{a}\left(q, i \omega_{n}\right)\left(G_{0}^{-1}\right)_{\alpha \beta}^{a b}\left(q, i \omega_{n}\right) \phi_{\beta}^{b}\left(-q,-i \omega_{n}\right),
$$

where $\alpha, \beta=f, b$ while Latin indices run from 1 to $n$, the number of replicas. The free propagator is a $2 n \times 2 n$ matrix given by $\left(G_{0}^{-1}\right)_{\alpha \alpha}^{a b}\left(q, i \omega_{n}\right)=\delta_{a b} /\left(\pi K_{\alpha}\right)\left(\omega_{n}^{2} / v_{\alpha}+v_{\alpha} q^{2}\right)$ and $\left(G_{0}^{-1}\right)_{f b}^{a b}\left(q, i \omega_{n}\right)=\left(G_{0}^{-1}\right)_{b f}^{a b}\left(q, i \omega_{n}\right)=\delta_{a b} U_{b f} q^{2} / \pi^{2}$. The idea of the GVM is to replace the complicated action $S$ in replica space by its best Gaussian approximation, $S_{G}$, with $\left(G^{-1}\right)_{\alpha \alpha}^{a b}=\left(G_{0}^{-1}\right)_{\alpha \beta}^{a b}-\sigma_{\alpha \beta}^{a b}$, and $\sigma_{\alpha \beta}^{a b}$ the selfenergy. The optimal $G$ can then be obtained by minimizing the variational free energy, $F_{\mathrm{var}}=F_{G}+\left\langle S-S_{G}\right\rangle_{G} / \beta$ with respect to $G_{\alpha \beta}^{a b}$.

Similar to Ref. [27], we find that the phase with localized fermions and superfluid bosons is well described by assuming one step replica symmetry breaking (1RSB) in the fermionic sector [28]. We obtain a fermion mass $\hat{\Sigma}_{f}=\xi_{f}^{-2}$,

$$
\hat{\Sigma}_{f}=\left(\frac{K_{f}^{2}}{v_{f}^{2}} \frac{D_{f}}{\sqrt{1-g^{2}}} \frac{2}{\Lambda}\right)^{\frac{2}{3-X_{f}}} \Lambda^{2},
$$

in agreement with the RG, and the solution ceases to exist when $X_{f}>3$. An RG calculation with respect to the latter variational action $\left(S_{G}\right.$ with $\xi_{f}$ finite) provides a modified RG equation which indeed smoothly interpolates between the limiting cases in Eq. (11), and can be used to compute the localization length, $\xi_{b}$. In the intermediate region $\mathrm{BFG}^{*}$ we find an extremely large, but finite $\xi_{b}$ (see Fig. 2). Similarly, we can compute the superfluid stiffness $\mathcal{D}=\lim _{\omega \rightarrow 0} \lim _{q \rightarrow 0} \omega^{2} \overline{\left\langle\phi_{b}(q, \omega) \phi_{b}(-q,-\omega)\right\rangle}$ in the $A G+$ SFB phase using our variational solution. We find that $\mathcal{D}>0$ only if $K_{b}>3 / 2$, indicating a phase transition towards a Bose Glass phase. This BFG* phase for $K_{b}<3 / 2$ appears in the variational problem as a a level 2 replica-symmetry breaking solution (RSB), with the replica symmetry also broken in the bosonic sector. However, $\xi_{b}$ being extremely large in the $\mathrm{BFG}^{*}$ region, it may appear as a superfluid phase in a finite system [28]. The nature of the transition between the BFG and the LL is quite subtle and will be detailed in [28.

Observables. The dynamical response of the Bose-Fermi mixture varies from one phase to another. Such a response can be probed through Bragg scattering measurements (see e.g. [29]), giving access to the dynamical structure factor $S_{b / f}(q, \omega)=$ $\int d t d x e^{i q x-i \omega t} \overline{\left\langle\rho_{b / f}(x, t) \rho_{b / f}(0,0)\right\rangle}$. The variational approach enables us to compute $S_{b / f}(q, \omega)$, at least for small values of $q$. Fig. 2 shows the inelastic part of $S_{f}(q, \omega)$ computed at two points of the phase diagram. The elastic part that comes from the gauge transformation used to absorb forward scattering would appear as a Dirac delta at $\omega=0$. The main plot shows the fermion structure factor in the BFG phase. It exhibits a two peak structure with peak locations $\omega \approx v_{b}\left(q^{2}+\hat{\Sigma}_{b}\right)^{1 / 2}$ and $\omega \approx v_{f}\left(q^{2}+\hat{\Sigma}_{f}\right)^{1 / 2}$. The first peak is a bosonic feature, indicating that the Bose-Fermi glass is indeed a coupled localized phase. The main effect of disor- 


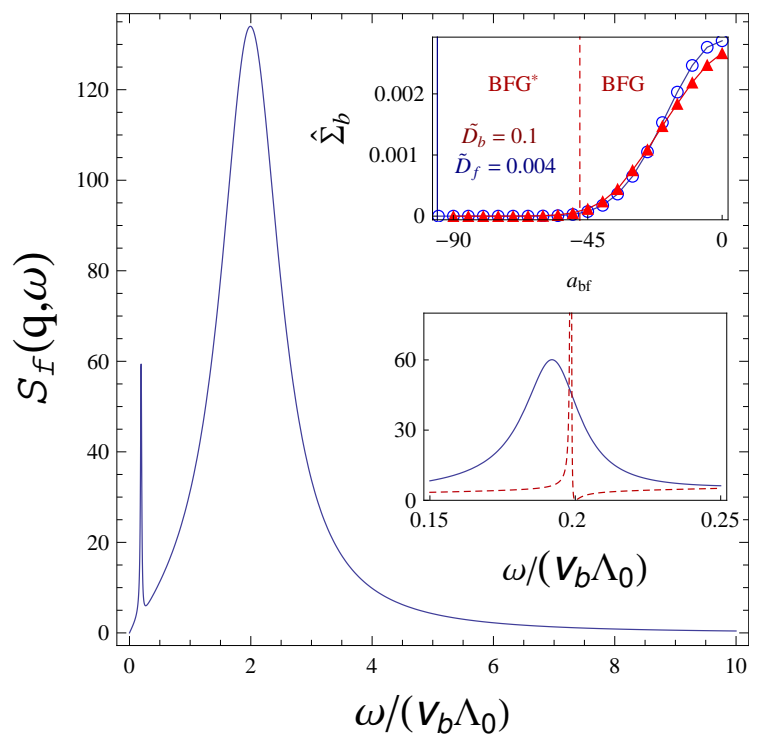

FIG. 2: Main plot: fermion structure factor $S_{f}\left(q=0.2 \Lambda_{0}, \omega\right)$ in the BFG phase as computed by GVM. Bose-Fermi scattering length is $a_{b f}=-35 a_{0}$. The UV cut-off is $\Lambda_{0}=\rho_{b}$. We have taken $V_{\perp, b}=40 E_{R, b}$. Other parameters are the ones of Fig. 1 Bottom inset is a zoom around the first peak at $\omega=v_{b}\left(q^{2}+\hat{\Sigma}_{b}\right)^{1 / 2}$, a signature of the coupled nature of the Bose-Fermi glass. The additional dashed curve is the structure factor in the $\mathrm{AG}+\mathrm{SFB}$ phase $\left(V_{\perp, b}=13 E_{R, b}\right)$. Top inset shows $\hat{\Sigma}_{b}=\xi_{b}^{-2}$, as obtained from the RG flow (circles) and the 2 -step $\mathrm{RSB}$ variational calculation (triangles). $V_{\perp, b}$ (i.e. $K_{b}$ ) is fixed and the boundaries obtained from the first RG analysis are represented. One can see that in region $\mathrm{BFG}^{*}$, the mass is non zero but extremely small. Below $a_{b f}=-95 a_{0}$ $\left(X_{f}=3\right), \hat{\Sigma}_{b}$ is identically zero.

der is to introduce new energy scales in the structure factor, $v_{f} \hat{\Sigma}_{f}^{1 / 2}$ and $v_{b} \hat{\Sigma}_{b}^{1 / 2}$. Its other effect is to introduce a linear frequency dependence for small $\omega$ [27, 28]. Notice that in the localized phases there is no hard gap in the excitation spectrum. The bottom inset is a zoom around the bosonic peak. Its counterpart in the $\mathrm{AG}+\mathrm{SFB}$ phase is plotted there (for clarity it has been left out in the main plot). Note that in the AG+SFB $\hat{\Sigma}_{b}$ is zero and the peak at $\omega \sim v_{b} q$ is much sharper, as it would be in a simple Luttinger liquid. Finally, in the LL phase, where both species are free, one would simply find two very sharp peaks, corresponding to the two sound modes of the mixture, at $\omega=v_{+} q$ and $\omega=v_{-} q$, with $v_{ \pm}^{2}=\frac{1}{2}\left(v_{f}^{2}+v_{b}^{2}\right) \pm \frac{1}{2}\left(\left(v_{f}^{2}-v_{b}^{2}\right)^{2}+4 g^{2} v_{f}^{2} v_{b}^{2}\right)^{1 / 2}$ [21, 22].

The transition from the $\mathrm{AG}+\mathrm{SFB}$ state to the $\mathrm{BFG}$ phase can easily be detected through time of flight (TOF) measurements. In the $\mathrm{AG}+\mathrm{SFB}$ phase one has $\left\langle\psi_{b}(x) \psi_{b}^{\dagger}\left(x^{\prime}\right)\right\rangle \sim 1 /\left|x-x^{\prime}\right|^{1 / 2 K_{b}^{*}}$, with $K_{b}^{*}$ the renormalized Luttinger parameter of the bosons. As a result, a bosonic coherence peak is predicted with a power law dependence, $n_{b}(R) \sim 1 / R^{1-1 / 2 K_{b}^{*}}$, with $n_{b}$ the density of bosons as measured in the TOF experiment, and $R$ the expansion along the 1D tubes.

Conclusion To summarize, we have established the phase diagram of an interacting Bose-Fermi mixture in the presence of uncorrelated disorder. For relevant experimental parameters, in the case of a ${ }^{87} \mathrm{Rb}-{ }^{40} \mathrm{~K}$ mixture, we have found three different phases, including a BoseFermi glass where both species are coupled and localized. These new phases can be detected by Bragg scattering and time of flight measurements.

Acknowledgment. We acknowledge fruitful discussions with N. Laflorencie. This work has been partially supported by the Institut Universitaire de France, the OTKA Grant No. K73361, and the CNCSIS grant No. ID672/2009.

[1] I. Bloch, J. Dalibard, and W. Zwerger, Rev. Mod. Phys. 80, 885 (2008).

[2] M. Greiner et al., Nature 415, 39 (2002).

[3] T. Stöferle, H. Moritz, C. Schori, M. Kohl, and T. Esslinger, Phys. Rev. Lett. 92, 130403 (2004).

[4] U. Schneider, et al., Science, 322, 1520 (2008).

[5] G. B. Partridge et al., Science 311, 503 (2006).

[6] M. W. Zwierlein, A. Schirotzek, C. H. Schunck, and W. Ketterle, Science 311, 492 (2006).

[7] F. Schreck, et al., Phys. Rev. Lett. 87, 080403 (2001) .

[8] Z. Hadzibabic, et al., Phys. Rev. Lett. 88, 160401 (2002).

[9] K. Günter, T. Stöferle, H. Moritz, M. Köhl, and T. Esslinger Phys. Rev. Lett. 96, 180402 (2006).

[10] S. Ospelkaus, et al., Phys. Rev. Lett. 96, 180403 (2006).

[11] T. Best, et al., Phys. Rev. Lett. 102, 030408 (2009).

[12] Ottenstein et al., Phys. Rev. Lett. 101, 203202 (2008).

[13] C. Honerkamp and W. Hofstetter, Phys. Rev. Lett. 92, 170403 (2004).

[14] A. Rapp, G. Zaránd, C. Honerkamp, and W. Hofstetter, Phys. Rev. Lett. 98, 160405 (2007)

[15] J. Billy, et al., Nature 453, 891 (2008).

[16] G. Roati, et al., Nature 453, 895 (2008).

[17] Th. Giamarchi and H. J. Schulz, Phys. Rev. B 37, 325 (1988).

[18] M. P. A. Fisher, P. B. Weichman, G. Grinstein, and D. S. Fisher, Phys. Rev. B 40, 546 (1989).

[19] L. Fallani, J. E. Lye, V. Guarrera, C. Fort, and M. Inguscio, Phys. Rev. Lett. 98, 130404 (2007).

[20] V. Ahufinger et al., Phys. Rev. A 72, 063616 (2005).

[21] M. A. Cazalilla and A. F. Ho, Phys. Rev. Lett. 91, 150403 (2003).

[22] L. Mathey, D.-W. Wang, W. Hofstetter, M. D. Lukin, and E. Demler, Phys. Rev. Lett. 93, 120404 (2004).

[23] F. Hebert, F. Haudin, L. Pollet, and G. G. Batrouni, Phys. Rev. A 76, 043619 (2007);

[24] F. D. M. Haldane, Phys. Rev. Lett. 47, 1840 (1981).

[25] M. A. Cazalilla, J. Phys. B: At. Mol. Opt. Phys. 37, S1S47 (2004).

[26] M. Mézard, G. Parisi, J. Phys. I 1, 809 (1991)

[27] T. Giamarchi and P. Le Doussal, Phys. Rev. B 53, 15206 (1996)

[28] F. Crépin, G. Zaránd and P. Simon, in preparation.

[29] D. Clement, N. Fabbri, L. Fallani, C. Fort, and M. Inguscio, Phys. Rev. Lett. 102, 155301 (2009).

[30] See EPAPS Document No. [] for the correspondence between experimental parame- 
ters $V_{\perp, b}, \quad a_{b f}$ and the Luttinger parameters $K_{b}, K_{f}, v_{b}, v_{f}$. For more information on EPAPS, see http://www.aip.org/pubservs/epaps.html.

[31] Overlining a quantity denotes disorder average.
[32] Integrating out high momentum fermions (bosons) also generates $V_{b}\left(V_{f}\right)$ through $U_{b / f}$, even if the disorder couples only to fermions (bosons). 